most of the economic problems confronting the medical profession, and many ethical ones which always arise under the temptations of poverty, would be solved, pirovided, of course, that there were no corresponding decrease in the demand for medical services.

How slowly any such ratio as 1 to 1,000 can be reached under any conceivable practical conditions may be illustrated as follows: If the rate of increase of total population falls only 2 per cent. more, following the analogy of the decades since 1880 , we shall have something like 108,000,000 inhabitants in 1920. The annual mortality of the medical profession in 1910 will be about 3,234. More than this number of physicians was graduated in 1880 and it is scarcely possible that, even by continuing to raise the medical standards, coalescing colleges and discouraging matriculants, the average graduation list can be still further diminished so as merely to keep the profession at its present numeric strength. But suppose that this result could be reached; the average clientele would be increased by only about 10 annually or by direct division of the expected population number in 1920 by the present estimated number of physicians the clientele at that time would be almost exactly 700 .

Allowing for a decennial increase of population dropping by 2 per cent. for each decade, the medical clientele would reach 811 in 1930 and 925 in 1940. In other words, we are about thirty-five years in advance of the requirements. This means not only individual average hardship for the medical profession, but a serious economic problem for the whole country.

\section{Clintcal Notes}

\section{A MICRO-ORGANISM WHICH APPARENTIY HAS A SPECIFIC RELATIONSHIP TO ROCKY MOUNTAIN SPOTTED FEVER}

\section{A PRELIMINARY REPORT * \\ H. T. RICKETTS, M.D. \\ CHICAGO}

Since the spring and summer of 1906 , bodies which I have referred to in my notes as "diplococcoid bodies," and sometimes short bacillary forms, have been found with considerable constancy in the blood of guinea-pigs and monkeys which were infected with Rocky Mountain spotted fever. They have also been seen in the blood of man but not so frequently. Much more time has been spent on the blood of the experimental animals than on that of man in view of the fact that it could always be obtained in fresh condition.

The form most commonly found is that of two somewhat lanceolate chromatin-staining bodies, separated by a slight amount of eosin-staining substance. The preparations of Giemsa, as furnished by Grübler, has been used almost exclusively, and with variations in the technic the intermediate substance may stain faintly blue.

In spite of the constancy with which these bodies were found, it did not seem justifiable to claim that they represent the microparasite of the disease, for two reasons: (1) the very complex morphology of the blood, especially in febrile states, when various cells and

\footnotetext{
* From the Pathological Laboratory of the University of Chicago
}

probably their nuclei are subject to unusual disintegration; (2) because of my inability to cultivate a microorganism of this character from infeeted blood by the use of ordinary and some unusual culture media, under various conditions of cultivation, or by other means to obtain it in satisfactory concentration. ${ }^{1}$

\section{THE BACILLUS IN THE TICK}

Although infected ticks had been examined previously in a more or less cursory manner, their systematic study was not undertaken until recently. In the pursuit of this work advantage was taken of the fact that the disease is transmitted by the infected female to her young through the eggs, as described in a previous report. A repetition of these experiments in the winter of 190\%-8, with the help of Dr. Maria B. Maver, resulted in such transmission in 50 per cent. of the ticks used, the fact being determined by allowing the larvæ to feed on normal guinea-pigs. This second series has not been published heretofore.

Female tick No. 40, a Dermacentor, from Montana, had produced fatal infections of spotted fever in guineapigs 1740 and 1764 . A number of eggs from the first. day's laying were crushed individually on cover glasses, fixed in absolute alcohol, and stained with Giemsa's stain. Each egg was found to be laden with astonishing numbers of an organism which appears typically as a bipolar staining bacillus of minute size, approximating that of the influenza bacillus, although definite measurements have not yet been made. Various forms are seen depending on the stage of development and the arrangement in which two or more may be found. It is very common to find two organisms end to end, with their poles stained deeply and the intermediate substance a faint blue, resembling a chain of four cocci. When the chromatin is not yet sharply limited to the poles, the somewhat lanceolate forms so often recognized in the blood are seen. Not infrequently. delicate bacilli with a uniform distribution of the chromatin are found. These are all interpreted as stages in the evolution of a bipolar organism. They are present in varying numbers in different eggs, but as a rule they are surprisingly numerous, and in some instances they would certainly count into the thousands. Many faintly staining, apparently degenerate forms are encountered.

Examination of the eggs of three dermacentors from Idaho (different specifically from the Montana dermacentor), which were infected from the guinea-pig, showed the presence of the same forms (Ticks 5, $y$ and 9$)$.

Conference with zoologic scientists who have made a particular study of the structure of eggs brought out the fact that such bodies are not known as a constituent of the egg of any species of animal.

Although it has not yet been possible to examine the eggs of ticks which are known to be free from spotted fever, the equivalent control has been made through a comparison of the visceral oroans of infected and uninfected ticks. The salivary glands, alimentary sac and ovaries of infected females are literally swarming with exactly similar micro-organisms. On the contrary, they appear to be entirely absent from the viscera of the uninfected tick, both male and female.

\section{AGGLUTINATION REACTIONS}

The most striking evidence of the probable etiologic relationship of this organism to spotted fever is found

1. Mr. P. G. Heinemann assisted in an extensive series of culture experiments in the spring of 1907 . 
in the positive outcome of agglutination tests. Fortunately it is so numerous in the eggs that a bacterial emulsion of reasonable concentration for agglutination tests can be made by crushing forty to fifty eggs in about 0.05 c.c. of salt solution. The material is so scant that only the microscopic method could be used. The preparations were made as hanging drops, incubated for two hours, dried, fixed with absolute alcohol and stained.

The serum of the normal guinea-pig either causes no agglutination at all, or at the most produces only slight agglutination in proportions of 1 to 1 and 1 to 9 . Dilutions higher than this cause no agglutination. In testing the agglutinating powers of immune serums, three animals which had, been infected from different sources and had recovered were used. One (1751) had been infected with a dermacentor from Idaho; another (1692), with a strain handed down direct from guineapig to guinea-pig for nearly three years without the intervention of ticks, the original infection having been obtained from the blood of man; the third (1757) with a strain kept in the same way since last spring. Graded dilutions, beginning with 1 to 1 and going as high as 1 to 200 , were used in the different series, with the striking result that a complete agglutinating power was present in the three immune serums in dilutions up to 1 to 160. It was somewhat less in a dilution of 1 to 200 . The highest dilution which will cause clumping has not been ascertained.

No fresh immune serum from man is at hand, but tests were made with three specimens which are about five, seven and nine months old respectively. They have been preserved in the ice chest with the addition of 0.3 per cent. of chloroform. The peculiar phenomenon of failure to agglutinate in concentrated solution was noted with all three. With the oldest serum no agglutination occurred until the dilutions of 1 to 160 and 1 to 200 were reached, when incomplete clumping was produced. With the second there was no agglutination in the dilution of 1 to 1 , distinct clumping in 1 to 10,1 to 20 and 1 to 40 , with little or none in bigher dilutions. In the serum of five months standing the reaction was absent in the dilutions of 1 to 1,1 to 10 and 1 to 20 , positive but not complete in 1 to 40,1 to 80 and 1 to 120 , with very little clumping in 1 to 160 and 1 to 200 . Normal human serum caused clumping in a dilution of 1 to 1 , a very slight amount in 1 to 10 , and none at all in the higher dilutions.

The failure of the immune serums from man to agglutinate in concentrated solutions, whereas they did so in higher dilutions, is taken as an example of the action of the so-called proagglutinoids. As explained by Ehrlich's theory, this consists in the occupation of the bacterial receptors by inactive agglutinin which exceeds the active agglutinin in its affinity for the bacteria. With higher dilutions of the serum the agglutinoids are so diluted that they do not completely occupy the bacterial receptors, thus affording a point of attack for the active agglutinin, unless the latter has been eliminated by extreme dilution. This is well known as a property of old agglutinating serums.

\section{BACILLI IN INFECTED SERUM}

As a means of concentrating the organisms in the serum of the infected guinea-pig the following experiment was performed: Three cubic centimeters of fresh infected serum were diluted with an equal amount of salt solution, and to this was added 0.3 c.c. of an agglu- tinating serum from the guinea-pig. The mixture was placed in the incubator for two hours and then centrifugated for about ten hours at a speed of 1800 revolutions. All but the merest drop was theli pipetted off and stained preparations were made of the sediment. Examination showed the presence of a moderate number of forms which are identical in appearance and size with those often recognized in ordinary smears of infected blood, and also with the "diplococcoid" forms seen in the egg of the infected tick. No such bodies were found in a control tube of normal serum.

The evidence pointing to this organism as the causative agent in spotted fever, though not complete, is of a striking character. In so far as I know it would be an unheard-of circumstance to obtain such strong agglutination with an immune serum, in the presence of negative controls, unless there were a specific relationship between the organism and the disease. In favor of the specific relationship in this case are also the presence of the organism in large numbers in infected ticks and in their eggs, its absence from uninfected ticks, and the presence of similar forms in the blood and serum of the infected guinea-pig.

Morphologically the organism is a bacillus and somewhat pleomorphic as described. Its resemblance to the bacilli of the hemorrhagic septicemias is striking, and in this connection it is important to note that spotted fever is a hemorrhagic septicemia. It has not been cultivated, although work with this end in view is in progress.

I have devised no formal name for the organism discussed, but it may be referred to tentatively as the bacilIus of Rocky' Mountain spotted fever. A further stuly of its characteristics may suggest a suitable name.

That a bacillus may be the causative agent of a disease in which an insect carrier plays an obligate rôle under natural conditions may be looked at with suspicion in some quarters. Yet, even without the evidence in this case, it would seem to be unscientific to be tied to the more or less prevailing belief that all such diseases must, on the basis of several analogies, be caused by parasites which are protozoon in character.

Further study of the relationship of the bacillus to the disease is being carried on and will be reported at a future date, together with illustrations and a more detailed account of its characteristics.

\section{A CASE OF FORMALIN POISONING}

\section{o. P. HUMPSTONE, M.D., AND WILLTAM LINTZ, M.D. BROOKLYN}

History.-Mrs. E. E., aged 33, was admitted to private ward of the Jewish Hospital, Oct. 16, 1907, suffering fiom ulcerative colitis, and was treated for eighteen days by high rectal irrigations of silver nitrate 1 to 1,000 solution. By mistake, on October 27, at 8 a. m., two ounces of formalin solution to a pint of water (about 12.5 per cent. strength) was usei for a rectal irrigation. After receiving this solution the patient cried out that it felt as though a red hot iron had been put in the rectum and that she was dying.

Symptoms.-The pain in the rectum and abdomen was excrueiating. There was marked pallor and coolness of the skin and mucus membranes, the face was expressionless. The eyes were dull and staring; the pupils were widely dilated and reacted very little. The heart action was delayed, irregular and feeble, the pulse being hardly perceptible. The respirations were irregular and sighing; deep breaths alternating with shallow inspiratory efforts. The mind was dull; cerebration was slow and questions were answered tardily and unwillingly. 